\title{
HISTORIA DE LOS ESTUDIOS PALEONTOLÓGICOS EN EL DEVÓNICO DE LA REGIÓN DE ALMADÉN (ZONA CENTROIBÉRICA, ESPAÑA): PERÍODO 1834-1990
}

\author{
Miguel V. PARDO ALONSO' \\ y Rodolfo GOZALO GUTIÉRREZ
}

Departamento de Geología. Universitat de València; Dr. Moliner, 50. E-46100 Burjassot, València. 'c/e: Miguel.V.Pardo@uv.es 2c/e: rodolfo.gozalo@uv.es

\begin{abstract}
Pardo Alonso, M.V. y Gozalo Gutiérrez, R. 1999. Historia de los estudios paleontológicos en el Devónico de la región de Almadén (zona Centroibérica, España): período 1834-1990.[History of the Devonian palaeontological research in Almadén region (Central-Iberian Zone, Spain): 1834-1990 period.] Revista Española de Paleontología, $\mathbf{n}^{\mathbf{0}}$ extr. Homenaje al Prof. J. Truyols, 217-228. ISSN 0213-6937.
\end{abstract}

\begin{abstract}
The first reference to fossils in the Almadén region occurs in Le Play (1834), whereas Ezquerra del Bayo (1838) was the first to refer to fossils that are unequivocally Devonian, even though "Devonian rocks" were mentioned for this region alredy in 1850. This historical study covers the 1834-1990 period and is based on a recopilation and analysis of the bibliography with regard to the palaeontology and stratigraphy of the Devonian of the Almadén region. In combination with fieldwork, this has allowed putting the classic palaeontological sites on the map, even in those cases where the locaties were previously unknown or of incertain location. As a result of our analysis of the various species mentioned, the Devonian palaeontological research in the region has been divided into seven periods, viz.: The initial period of time (1834-1873), The reorganization of the Comisión del Mapa Geológico (1873-1910), The early part of the 20th century (1910-1936), The second part of the 20th century (1936-1960), The German school and Almela et al.'s monographic study (1960-1970), Research on mineral deposits (1970-1980), and The reactivation of palaeontological research in relation to the MAGNA geological mapping project (1980-1990).
\end{abstract}

Keywords: History of Palaeontology, Devonian, Central-Iberian Zone, Spain.

\section{RESUMEN}

Las primeras citas de fósiles en la región de Almadén se remontan al trabajo de Le Play (1834), siendo Ezquerra del Bayo (1838) el primero en citar fósiles inequívocamente devónicos, si bien no es hasta 1850 cuando se habla propiamente de "terrenos devónicos". El presente estudio histórico, que abarca el período 1834-1990, se ha basado en una recopilación bibliográfica de las obras que han versado sobre paleontología y estratigrafía del Devónico de la región de Almadén y su posterior análisis. Éste, junto con un detallado trabajo de campo, ha permitido la elaboración de un mapa de situación de los yacimientos clásicos presentes en el área, muchos de ellos de localización incierta o desconocida hasta la fecha. Por último, en función de las citas de especies de cada trabajo analizado, se ha procedido a la delimitación y caracterización de siete períodos en el conocimiento de la región: Primera Época (1834-1873), Reorganización de la Comisión del Mapa Geológico (1873-1910), Primer tercio de siglo (1910-1936), Segundo tercio de siglo (1936-1960), Escuela alemana y Monografía de Almela et al. (1960-1970), Estudios de yacimientos minerales (1970-1980), y Reactivación y Hojas MAGNA (1980-1990).

Palabras clave: Historia de la Paleontología, Devónico, Zona Centroibérica, España.

\section{INTRODUCCIÓN}

En los trabajos geológicos, particularmente desde las obras de Prado (1855) y Verneuil y Barrande (1855), se ha venido denominando como "región de Almadén" a un área situada en la parte media de la rama sur de la Zona Centroibérica (ZCI: Julivert et al., 1974). Esta área (Fig. 1 ), en su sentido más amplio, incluye los sinclinales de 
Herrera del Duque, Almadén, Guadalmez y parte del flanco norte del sinclinal de Pedroches, que geográficamente se sitúan en las estribaciones septentrionales de Sierra Morena, en tránsito hacia los Montes de Toledo.

En los núcleos de estos sinclinales afloran materiales del Devónico, cuyo contenido fosilífero, conocido desde el primer tercio del siglo XIX, ha merecido desigual atención a lo largo del tiempo. La importancia minera del establecimiento de Almadén, que contribuyó al impulso inicial de este conocimiento, hizo que los principales estudios posteriores se centraran en la investigación minera, en detrimento de los estudios paleontológicos y estratigráficos.

Este desinterés contrasta con la importancia que tiene esta región, en cuanto al conocimiento de los materiales y contenido fosilífero del Devónico de la ZCI, ya que esta franja meridional, junto al extremo nororiental (sinclinal de Alcañices), son los únicos lugares de la ZCI (en su concepción clásica) en los que se ha conservado registro de materiales devónicos. Además, la región de Almadén es la que presenta menor complejidad tectónica dentro de la franja meridional y, sobre todo, posee las series estratigráficas y el contenido paleontológico más completos; se debe destacar también que 24 especies y una subespecie devónicas se han descrito originalmente a partir de material recogido en la región, donde tienen su localidad tipo.

\section{LA INVESTIGACIÓN PALEONTOLÓGICA EN LA REGIÓN}

El presente análisis tiene su origen en la revisión de los antecedentes bibliográficos sobre el tema de Tesis Doctoral de uno de nosotros (Pardo Alonso, 1997). El período investigado se inicia en 1834 , año en que se publica el trabajo de Le Play, que contiene la referencia más antigua que se ha encontrado sobre la existencia de fósiles en la región; el análisis alcanza hasta 1990, año en que se publicó la última Hoja del MAGNA editada hasta el momento que incluye parte de la región, y se inicia el trabajo de investigación de la citada tesis. El tipo de búsqueda bibliográfica utilizada ha consistido en un vaciado de las referencias citadas en fuentes secundarias, esto es, una vez localizados algunos de los trabajos que hacían referencia al área y período elegidos, se ha procedido al vaciado de su bibliografía y así de manera sucesiva, hasta obtener una recopilación de la mayoría de los trabajos que han versado sobre el área y cuya temática principal era de tipo paleontológico, estratigráfico o de geología regional.

Dentro de este intervalo temporal, los trabajos paleontológicos sobre el Devónico de la región casi siempre han acompañado a los estudios estratigráficos y/o mineros, a los que han servido como mero complemento, limitándose en la mayoría de los casos a listados de fósiles. Pocos son los trabajos que se pueden considerar realmente estudios paleontológicos, ya que únicamente cuatro de ellos incluyen descripciones originales de fósiles devónicos encontrados en la región, y sólo uno de ellos lo hace con cierta profundidad.

Así, las temáticas paleontológicas tratadas en los trabajos de investigación sobre el Devónico de la región han sido principalmente la identificación de taxones fósiles y, en menor medida, la descripción y figuración de los mismos. Son escasos los que abordan la distribución estratigráfica de los taxones y biozonación, y, ocasionalmente y de un modo muy somero, se han realizado consideraciones paleobiogeográficas y paleoambientales.

Por otro lado, desde el punto de vista de la investigación puramente geológica, los datos paleontológicos han sido necesarios en muchos casos, debido a la complejidad tectónica de la zona, la escasez y discontinuidad de los afloramientos, y la monotonía de las series. En este apartado, la investigación paleontológica ha sido decisiva en la identificación de los niveles pertenecientes al sistema Devónico y de sus unidades cronoestratigráficas menores (series y pisos), conocimiento de las relaciones entre las capas devónicas y los niveles de los sistemas Silúrico y Carbonífero, división litoestratigráfica de los niveles devónicos, identificación de las discontinuidades en el registro (notablemente la laguna del Devónico Medio, característica de la ZCI meridional y Zona Ossa-Morena), cartografía geológica e interpretación estructural de los sinclinales donde aflora el Devónico en la región. Muy puntualmente los datos paleontológicos han permitido nuevas precisiones al conocimiento de los materiales volcánicos que afloran en el núcleo del sinclinal de Almadén.

\section{GEOGRAFÍA DE LOS YACIMIENTOS CLÁSICOS DE LA REGIÓN DE ALMADÉN}

Los trabajos que habitualmente se consideran clásicos son los anteriores a la monografía de Almela et al. (1962); en ellos aparecieron citadas una serie de localidades fosilíferas que, por extensión, también se denominan "clásicas". Resulta interesante conocer la posición actual de dichas localidades (Fig. 2), no sólo por el carácter histórico, sino por ser varias de ellas localidades tipo de alguna de las especies definidas en el área.

En los primeros trabajos se alude a localidades fosilíferas de un modo muy general e indeterminado, siendo Verneuil (en Anónimo, 1850) el primero en citar de un modo preciso yacimientos fosilíferos del Devónico en la región: Cerro de Castillejo, Puerto del Ciervo y Casa de la Vega; a éstos añadió Luján (1851) el de las "calizas del Enjambradero" al norte de Almadén. Un poco más adelante, Prado (1855) y Verneuil y Barrande (1855) dieron a conocer 13 nuevas localidades que, junto con las citadas antes por Verneuil, constituyen casi las dos terceras partes de los 21 yacimientos que hoy se consideran clásicos en la región. Los yacimientos descubiertos por estos tres autores han sido 


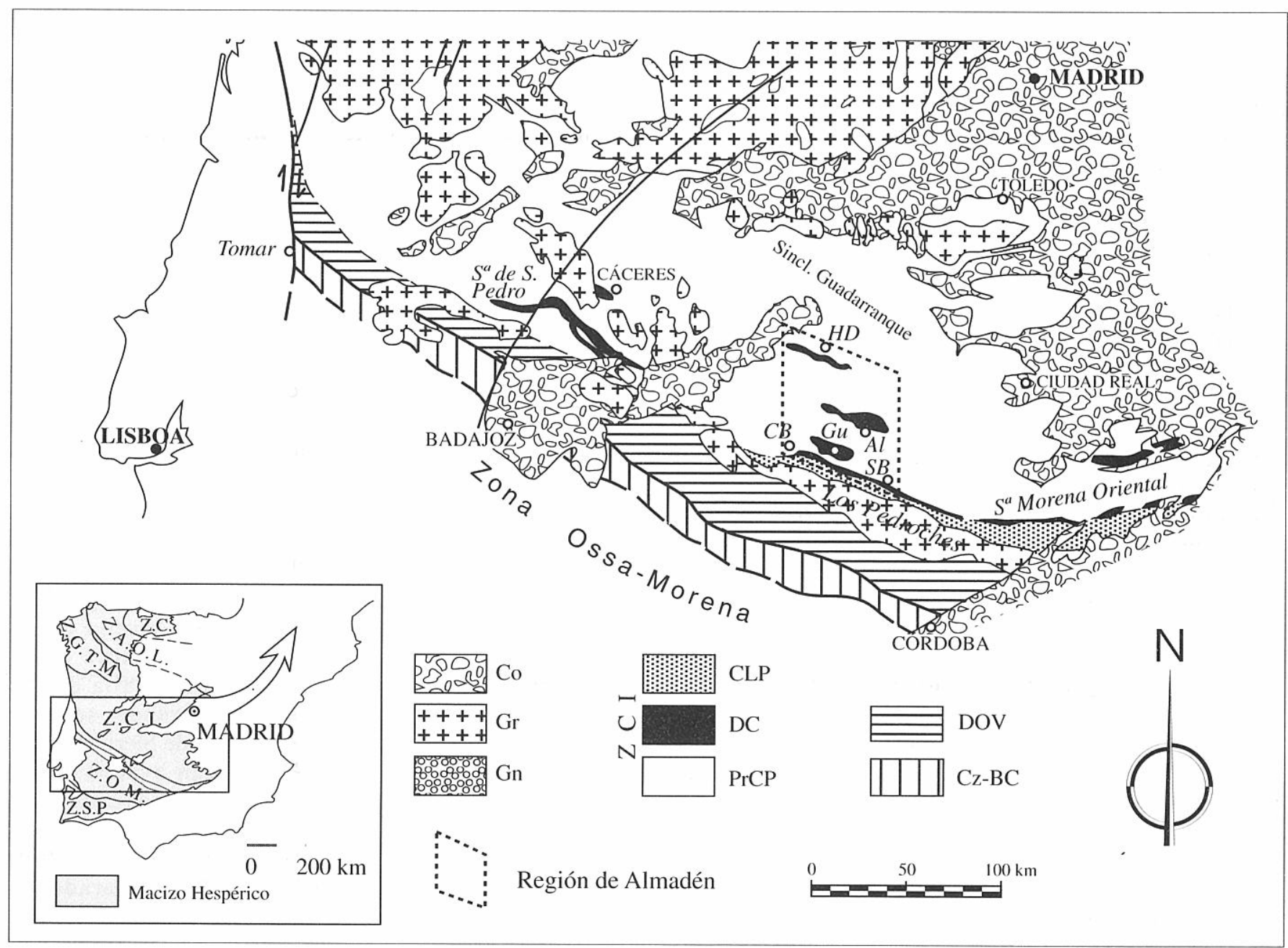

Figura 1. Situación geográfica y geológica de la región de Almadén. Abajo izquierda: Zonas del Macizo Hespérico y localización del área arriba ampliada. Arriba, mapa esquemático de la parte meridional de la Zona Centroibérica, con indicación de la región de Almadén (Modificado de Díez Balda et al., 1990, y Pardo Alonso y García-Alcalde, 1996).

Leyenda.- CLP: Carbonífero de Los Pedroches (norte); Co: Cobertera post-paleozoica; Cz-BC: Cizalla BadajozCórdoba; DC: Devónico (y Carbonífero de Guadalmez y Cáceres); DOV: Dominio de Obejo-Valsequillo; Gn: Gneises glandulares (Ollo de Sapo); Gr: Granitos hercínicos; PrCP: Precámbrico y Paleozoico pre-Devónico. Z.C.: Zona Cantábrica; Z.A.O.L.: Zona Astur Occidental-Leonesa; Z.G.T.M.: Zona Galicia Tras-Os-Montes; Z.C.I.: Zona Centroibérica; Z.O.M.: Zona Ossa-Morena; Z.S.P.: Zona Sur-Portuguesa.

Localidades.- Al: Almadén; CB: Cabeza del Buey; Gu: Guadalmez; HD: Herrera del Duque; SB: San Benito.

frecuentemente repetidos en obras posteriores, añadiéndose ocasionalmente algunas localidades nuevas; es el caso de Mallada (1880: Rinconcillo de Belalcázar y Cumbres de Santa Eufemia), Groth (1914a: Guadalmez), y Ciry (1936: La Calera, aunque posiblemente sea equivalente al que Prado cita como "Castillejo").

En conjunto, las localidades fosilíferas devónicas del ámbito de Almadén consideradas como clásicas son las siguientes, tal y como fueron citadas por primera vez:

Calizas del Enjambradero; Herrera del Duque (1); Guadalperal; Castillejo, entre Almadén y Almadenejos; Chillón; Viña de Ayllón; Casa de la Vega (2); alrededores de Almadén; Vallenegrillo; cerca de Almadenejos; Puerto del Ciervo, al norte de Almadén; Val mayor, una legua de Fuenlabrada, cerca de Herrera del Duque (1); una legua al S de Almadén (2); Cercones de Carbadillo; Cabeza del Buey (3); Convento de Chillón; Fuenlabrada (1); Cerro del Hinojo; Rinconcillo de Belalcázar (3); Cumbres de Santa Eufemia (3); Guadalmez (2).

Localidades situadas en: (1) sinclinal de Herrera del Duque; (2) sinclinal de Guadalmez; (3) flanco norte del sinclinal de Pedroches; las restantes corresponden al sinclinal de Almadén.

La mayoría de estos yacimientos clásicos fueron reencontrados por nosotros sin dificultad, sea a través de las descripciones y mapas originales, sea por tradición oral entre las personas relacionadas con la minería de la zona; otros, sin localización precisa, pudieron encontrarse recientemente gracias a que el topónimo se conserva en 
los mapa actuales ("calizas del Enjambradero" o los "cercones de Carbadillo"); estos topónimos corresponden a áreas no muy extensas en las que existen yacimientos paleontológicos, cuyo contenido coincide en gran parte con el de los listados clásicos. Un caso especial es el del yacimiento de "Casa de la Vega", que se daba por perdido a pesar de figurar en el mapa de Prado (1855), debido a imprecisiones en el dibujo de la topografía y por la ausencia del topónimo en los mapas actuales; sin embargo, se ha podido identificar su posición aproximada al haberse conservado el topónimo entre los habitantes del lugar.

Unas pocas localidades no han podido ser situadas por no existir mapas y por lo ambiguo del topónimo aplicado a las mismas, generalmente en referencia a un área muy extensa. Son los casos, por ejemplo, de "Herrera del Duque" o "Cabeza del Buey", y otras localidades en cuyo entorno existen multitud de yacimientos fosilíferos de esta edad. Quedan también sin localizar varios yacimientos no reflejados en los mapas y cuyos topónimos se han perdido (como "Viña de Ayllón").

\section{HISTORIA DE LAS PRINCIPALES INVESTIGACIONES PALEONTOLÓGICAS EN LA REGIÓN}

La primera alusión paleontológica no se encuentra hasta el siglo XIX en que Le Play (1834), en un trabajo sobre la geología de Extremadura y norte de Andalucía, indicó la presencia de fósiles en Almadén y Santa Eufemia, entre otras localidades. Esta misma obra es notable también por encontrarse en ella el primer mapa geológico que incluye la región de Almadén.

La siguiente referencia está en Ezquerra del Bayo (1838), que resaltó la abundancia de restos orgánicos en los "terrenos más modernos de la grauvaca", donde citó la presencia, entre otras, de varias especies de braquiópodos y bivalvos en Almadén.

Entre los años 1840 y 1870 se registra el primer gran impulso en el conocimiento geológico de la región, en especial en paleontología y estratigrafía, con los trabajos del francés Edouard de Verneuil y colaboradores.

En el número I de la Revista Minera (1850), un autor anónimo publicó una nota sobre fósiles hallados por Verneuil en España, que constituyó un avance informal de las investigaciones paleontológicas de este autor. En un trabajo posterior, Verneuil y Collomb (1853) atribuyeron la autoría de esta nota anónima al propio Verneuil, hecho que asumieron también los autores de la época; el investigador francés mantenía una correspondencia fluida con varios ingenieros de minas españoles, algunos de ellos redactores de la Revista Minera, siendo éstos los que publicaban las cartas de Verneuil una vez traducidas. Aunque entre los fósiles citados anteriormente por Ezquerra había especies devónicas, fue en este trabajo donde por primera vez se mencionó expresamente el Devónico, identificado por su contenido paleontológico.

A la vez, Murchison (1850) presentó un primer esquema geológico de los depósitos sedimentarios de España, comunicado también por el propio Verneuil, donde se encuentra un listado de fósiles devónicos, entre otros; el listado en parte repite las citas de lo publicado en la Revista Minera, a las que se añadieron cuatro citas más.

De la misma época es el trabajo de Luján (1850, 1851 ), en cuya parte $2^{\mathrm{a}}$ el autor apuntó varias localidades con fósiles devónicos y proporcionó un mapa geológico del cuadrante suroccidental de España, en el que, para la región de Almadén, parece haber adaptado los datos del ya citado mapa de Le Play (1834).

En un trabajo de síntesis posterior, sobre la constitución geológica de algunas provincias de España, Verneuil y Collomb (1853) vuelven a citar muy sucintamente el Devónico de Almadén, incluyendo un pequeño listado de fósiles devónicos, todos ellos ya citados con anterioridad.

Nuevas citas de fósiles devónicos de Almadén se deben a Ezquerra del Bayo (1854), aunque parte de las identificaciones son las publicadas en la Revista Minera y en Murchison (op. cit.). El mismo Ezquerra proporcionó en 1856 un mapa geológico de España, mucho menos detallado que los anteriores de Le Play y de Luján.

La publicación en 1855 de la monografía en dos partes, de Prado y de Verneuil y Barrande, sobre la geología de Almadén y zonas próximas de Sierra Morena y Montes de Toledo, supuso un gran avance en los estudios regionales y en general en la investigación geológica española. En la primera parte, Prado comienza plasmando sus observaciones sobre la geología de la región de Almadén, describiendo por separado los materiales devónicos y "silurianos" (Ordovícico+Silúrico en sentido actual, a partir de aquí el término "Siluriano" se emplea siempre en este sentido). Debido a errores de datación (falta de caracterización del "Siluriano superior" y asignación al Devónico de niveles calcáreos que corresponden en realidad al Ordovícico), Prado interpretó que los materiales devónicos yacían discordantes sobre el "Siluriano inferior". Aunque el estudio detallado de los fósiles fue abordado en la segunda parte de la monografía por Verneuil y Barrande, Prado aportó numerosos datos paleontológicos, incluyendo para el Devónico un listado de faunas fósiles y yacimientos agrupados por litologías y también una cartografía, desde el norte de Almadén hasta Santa Eufemia (Córdoba), en el que se diferencian, por primera vez, los terrenos devónicos de los "silurianos".

Verneuil y Barrande describieron y figuraron el material fosilífero recogido por el propio Verneuil, por Eusebio Sánchez (director de las minas de Almadenejos) y, principalmente, por Casiano de Prado. En este trabajo se encuentra el primer apunte paleobiogeográfico de la región, al señalar los autores la semejanza paleontológica de los terrenos paleozoicos españoles con los de Francia y Bohemia, y el contraste con los de Inglaterra, Suecia y Rusia; también destacaron la necesidad de utilizar los fósiles para reconstruir la estratigrafía, debido a la dificultad introducida por la deformación de las rocas. En el trabajo sistemático, además de citar 62 especies devónicas, Verneuil y Barrande realizaron las primeras descripciones y figuraciones de fósiles devónicos de la 


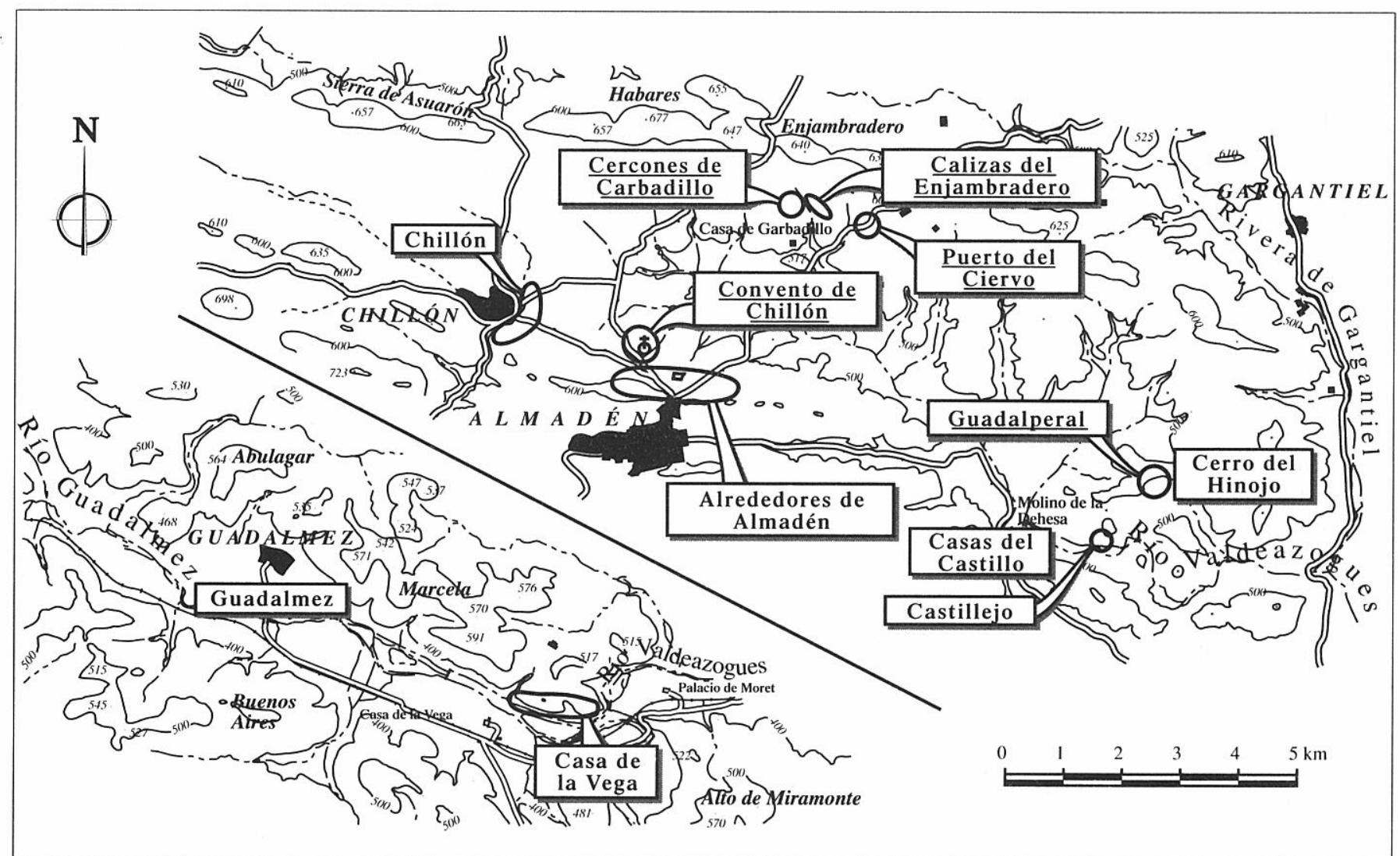

Figura 2. Mapa de yacimientos clásicos de fósiles devónicos en los sinclinales de Almadén (arriba derecha) y Guadalmez (abajo izquierda), cuya posición ha podido ser identificada. Se indican las localizaciones seguras (nombre subrayado), aproximadas (nombre con indicación del área posible) o dudosas dentro de una gran zona (nombre sin más indicación).

región, estableciendo ocho especies nuevas (aunque dos de ellas, las de corales, deben su descripción a Haimé). Los nuevos taxones fueron los bivalvos Avicula paillettei, Avicula leplayi, Avicula subcrinita y Avicula schulzii, el braquiópodo Terebratula mariana, el cefalópodo Cyrtoceras lujani, y los corales Acervularia pradoana y Combophyllum marianum.

Mallada (1875) describió someramente los materiales devónicos del área de Almadén, Santa Eufemia y Herrera del Duque, citando 57 especies de la zona, de las que describió 54 y redibujó algunas de las figuras de Verneuil y Barrande; esta obra fue complementada por su trabajo de 1892.

Gonzalo y Tarín (1879) aportó algunos datos nuevos sobre las rocas devónicas de Herrera del Duque, Garlitos y Cabeza del Buey; si bien los listados de fósiles son muy escasos, en el mapa geológico diferenció por vez primera los afloramientos devónicos de Herrera del Duque.

En 1880 aparecieron dos nuevos trabajos, debidos a Mallada y a Cortázar. Mallada citó, en la provincia de Córdoba, varios afloramientos nuevos de rocas devónicas con fósiles, uno de ellos cercano a Cabeza del Buey. Por su parte, Cortázar describió las rocas devónicas de la provincia de Ciudad Real, precisando las litologías predominantes, pero sin aportar grandes novedades respecto a los datos de Verneuil y Barrande. Sobre la estructura, Cortázar adoptó a grandes rasgos las ideas de
Prado, suponiendo un contacto discordante entre materiales devónicos y "silurianos".

Mallada (1898) sintetizó las principales aportaciones de la Comisión del Mapa Geológico sobre el Devónico y Carbonífero de España, reflejando el estado de conocimientos sobre el Devónico español, con una puesta al día de los datos sobre el área de Almadén. Por el carácter sintético de esta obra, se encuentran pocas novedades en ella, salvo actualizaciones de algunos nombres.

Drevermann (1909) dio cuenta de los fósiles devónicos que encontró durante una corta estancia en la zona de Almadén, separando por primera vez los yacimientos del Devónico Inferior y del Superior. Aunque no encontró fósiles del Devónico Medio, supuso su existencia por las citas anteriores de formas indicativas de esta serie. Este material fue posteriormente estudiado en Alemania por Müller y Paeckelmann.

Al margen de esta pequeña nota de Drevermann, es posible decir que con la síntesis de Mallada se termina una etapa, iniciándose con Groth otra nueva en la que se tiende a precisar la estratigrafía regional con más detalle. Este autor presentó, a partir de 1911, varias comunicaciones a la Academia de Ciencias de París, donde da cuenta de sus investigaciones en el ámbito de Sierra Morena.

En la primera de ellas, Groth (1911) elaboró el primer 
esquema estratigráfico del Devónico, centrado en el área de Guadalmez y proporcionó la primera interpretación sobre la evolución paleoambiental de la región durante este período. En su concepción estructural, se habla ya de los sinclinales devónicos de Guadalmez y Almadén, separados por un anticlinal de materiales "silurianos", y establece las relaciones de concordancia entre los primeros niveles que atribuye al Devónico y las "ampelitas de Monograptus", descartando implícitamente las interpretaciones anteriores.

En la siguiente comunicación, Groth (1914a) estudió en detalle niveles pelíticos que denominó "schistes à goniatites" de Guadalmez, citando varias especies de cefalópodos y bivalvos, con las que aseguró una edad Devónico Superior, muy probablemente Fameniense; el autor apuntó para las faunas centroibéricas un carácter "armoricano-varisco". En otro trabajo (Groth, 1914b), resumió la estratigrafía de la región, diferenciando en Almadén los niveles del Devónico Inferior de los del Superior, e incluyó un pequeño listado de fósiles de esta área. Por último, presentó un mapa 1:250.000 donde se singularizan los materiales devónicos y carboníferos de la zona estudiada (desde Almadén hasta Córdoba), así como las principales estructuras tectónicas existentes. El mismo mapa se reproduce en un trabajo ulterior (Groth, 1914c).

El XIV Congreso Internacional de Geología, realizado en Madrid en 1926, no aportó nada nuevo al conocimiento del Devónico de la zona por parte de los investigadores de la región, más interesados en los aspectos mineros. Únicamente se hace alusión a los fósiles devónicos en un listado elaborado por F. Barrande (in Hernández Sampelayo et al., 1926) incluido en la guía de la excursión B-1, que tuvo como objeto las Minas de Almadén; este listado, que comprende 90 especies y los yacimientos donde se encontraron, la mayoría del Devónico Inferior, se inspiraba en el de Mallada (1898), y no incluye los del Fameniense de Groth (1914a).

Müller (1929) estudió el material fosilífero del Frasniense, recogido años antes por Drevermann, y describió y figuró 34 especies de invertebrados, principalmente braquiópodos y bivalvos; entre estos últimos describió 9 especies nuevas: Carydium beushauseni, Cypricardella oblonga, Myophoria rotunda, Leptodesma almadenensis, Avicula? (Dolabra?) hispanica, Modiola supradevonica, Cucullella planiforma, Ctenodonta robustella y Nuculana acuta. Otro aspecto importante es la separación de cinco formaciones areniscosas de acuerdo con su contenido fosilífero, que será el germen de la biozonación establecida por Pardo y García-Alcalde (1984). La obra de Müller (op. cit.) junto con la de Verneuil y Barrande (1855) son las principales obras paleontológicas no recopilatorias realizadas en el área.

Ciry (1936), en un pequeño estudio geológico sobre la región de Almadén, completó el esquema estructural ya esbozado por Groth. El esquema estratigráfico es mucho más detallado y ordenado que los anteriores, estableciendo la autora el esquema básico de las grandes unidades litoestratigráficas actuales. De acuerdo con el contenido fosilífero, distinguió niveles de edad diferente dentro del Devónico Inferior, dató como Devónico Medio los niveles volcánicos frasnienses del núcleo del sinclinal, atribuyendo al Frasniense los materiales devónicos más jóvenes de este sinclinal.

A partir de 1954, se realizaron una serie de tesis doctorales sobre la geología regional de España y Portugal, por investigadores alemanes de la Universidad de Münster, dirigidas por Franz Lotze. Algunas zonas investigadas quedan dentro de los límites de nuestra zona de estudio y son, a veces, interesantes por incorporar las primeras sucesiones estratigráficas y cartografías detalladas de la región, aunque la mayoría quedaron inéditas.

Márquez Triguero (1961) fue el primero en citar fósiles devónicos en el extremo suroccidental de la región (San Benito), además aportó una cartografía de estos niveles, interpretados como parte del flanco norte del sinclinal de Los Pedroches.

El trabajo de Almela et al. (1962), que tuvo su precedente en el de Almela (1959), constituye un hito fundamental en el conocimiento del Paleozoico de la región. En el capítulo estratigráfico, distinguen por primera vez algunas unidades litológicas asimilables a formaciones, y comienzan la serie devónica en la llamada "Cuarcita de base" (aunque se admite que no representa estrictamente el comienzo del sistema). En cuanto a la Paleontología, los autores indicaron la posición y contenido fosilífero de 59 yacimientos devónicos; las determinaciones parecen mostrar una serie devónica completa, incluyendo materiales del Devónico Medio, aspecto que será rechazado por autores posteriores. En el apartado sistemático, se describen y figuran cinco especies del Devónico. La utilización exahustiva de datos paleontológicos permitió a los autores la realización de la primera cartografía geológica de detalle de la región.

Puschmann (1967a) publicó un trabajo que puso de manifiesto la existencia de una laguna estratigráfica en el Devónico de la región, desde la sierra de San Pedro hasta Fuencaliente, y que en el área de Almadén abarcaría todo el Devónico Medio. En el mismo año, Puschmann (1967b) estudió el área trabajada anteriormente por Márquez Triguero (op. cit.), modificando notablemente las dataciones de este autor, con nuevos datos paleontológicos que mostraron que la estratigrafía del Devónico era similar a la de las zonas próximas, incluyendo la laguna del Devónico Medio.

Puschmann (1970a) estudió la evolución paleogeográfica de la Península Ibérica durante el Devónico. En la zona de estudio, que incluye en la cuenca sedimentaria meridional, señaló la presencia de la "Facies tipo Renana", tanto en el Devónico Inferior como en el Superior. En otro trabajo, Puschmann (1970b) abordó el estudio del Paleozoico del sinclinal de Herrera del Duque; el Devónico lo dividió en ocho unidades litoestratigráficas y analizó su contenido paleontológico en detalle, alcanzando dataciones muy precisas; el estudio paleontológico comprende el primer tratamiento de conodontos de los niveles calcáreos del Devónico Inferior de la región.

Los restantes trabajos sobre la región realizados en la 


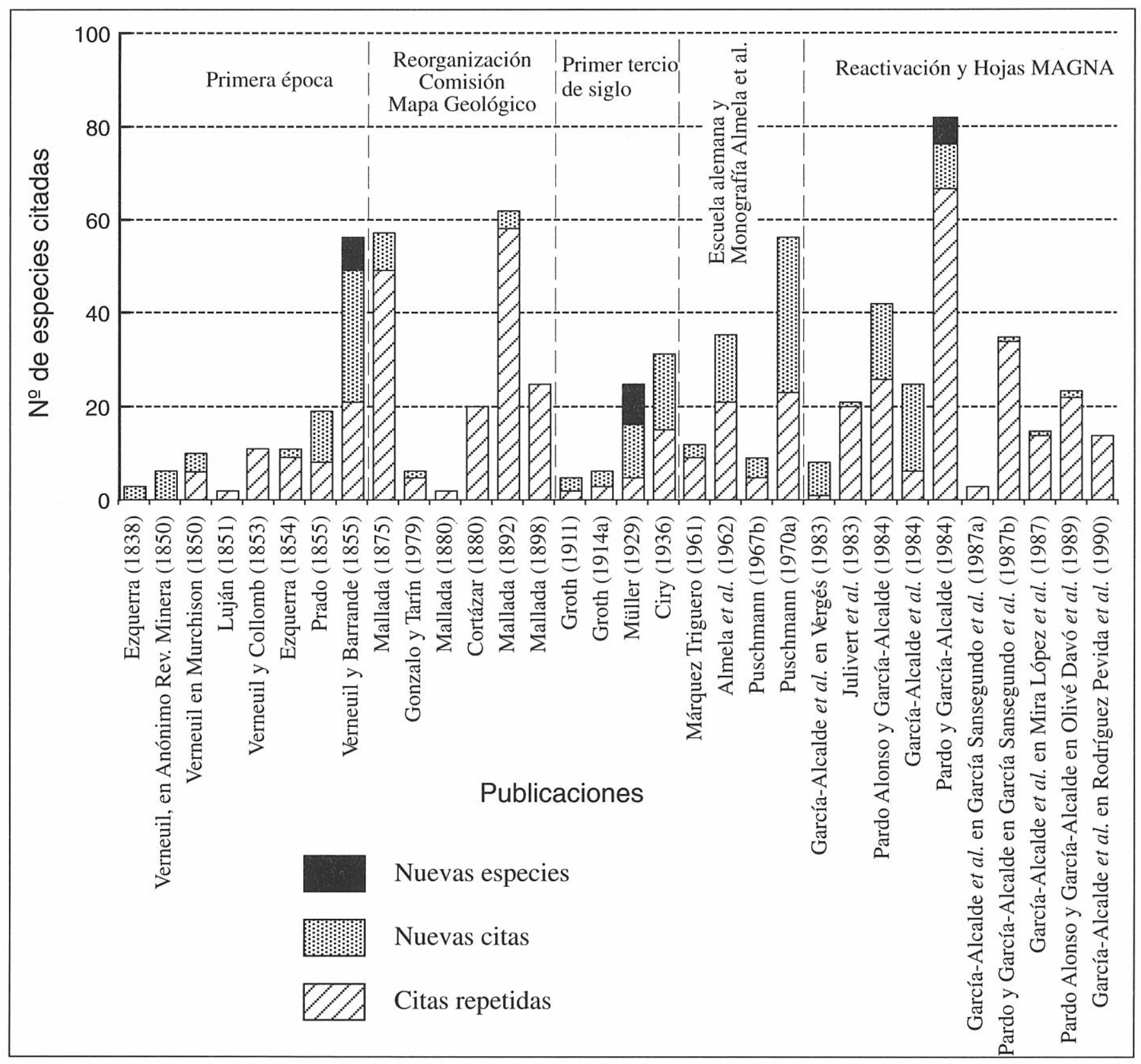

Figura 3. Distribución por publicaciones de las especies fósiles devónicas citadas en el área estudiada, hasta 1990.

década de 1970, se deben a varios autores franceses; son principalmente de índole minera, centrados en los yacimientos de mercurio y su entorno, y contienen breves referencias al Devónico. De ellos únicamente se publicó el de Saupé (1973), quedando los demás inéditos.

A comienzos de los años ochenta, resurgen los estudios puramente geológicos sobre la región, representados por tesis de licenciatura de índole estructural y petrológica, con aportaciones paleontológicas menores, centrados en los sinclinales de Almadén y Guadalmez.

Vergés (1983) estudió el complejo vulcanosedimentario del Devónico y la estructura de la terminación periclinal oriental del sinclinal de Almadén, aportando una cartografía, detallada sobre todo para los materiales volcánicos, y numerosos datos paleonto- lógicos, debidos a los Dres. J. L. García-Alcalde, M. A. Arbizu, S. García-López, F. Soto y M. Truyols-Massoni, de la Universidad de Oviedo.

Julivert et al. (1983) publicaron un trabajo sintético sobre el Devónico del Macizo Ibérico; dado su carácter recopilatorio, apenas se ofrecen datos nuevos sobre la región.

Pardo Alonso y García-Alcalde (1984) presentaron el primer esquema de división en unidades litoestratigráficas del Devónico de Almadén, ayudados por gran número de determinaciones paleontológicas de material recogido en numerosas localidades. Asimismo, realizaron el esbozo de una biozonación del Devónico Superior basada sobre todo en braquiópodos y bivalvos.

Pardo y García-Alcalde (1984) establecieron, para el Devónico del sinclinal de Almadén, dos grupos, uno 
detrítico y otro volcánico; dentro del Devónico Superior, distinguieron por primera vez dos unidades litoestratigráficas informales (Unidad 1 y Unidad 2) con rango de formación. También por primera vez en la región, se indica la distribución estratigráfica precisa de más de setenta taxones, reflejando la correspondiente a los treinta y tres más representativos en una columna estratigráfica que sintetiza todas las observaciones; estos datos permitieron acotar con más precisión la laguna estratigráfica mesodevónica en Almadén. Se estableció formalmente una biozonación del Frasniense, comprendiendo 5 biozonas de distinto rango, basadas en bivalvos y braquiópodos, muchas de las cuales tienen una amplia validez regional. Por último, los autores describieron y figuraron con detalle los principales taxones de braquiópodos frasnienses involucrados en la biozonación; entre ellos se encuentran 6 especies nuevas: Apousiella almadenensis, Cyphoterorhynchus domenechae, Pradochonetes muelleri, Douvillinoides ? alvarezi, Douvillina delta y Douvillina radiata, y un género nuevo: Pradochonetes. El trabajo se acompaña de un mapa de los tres sinclinales estudiados, donde situaron los más de setenta nuevos yacimientos fosilíferos encontrados.

García-Alcalde et al. (1984) estudiaron la problemática del límite Devónico-Carbonífero en la zona de Guadalmez-Santa Eufemia, estableciendo la estratigrafía precisa de los materiales situados en el entorno del límite, y un análisis detallado de los fósiles que contienen. Reinterpretaron gracias a ello la serie, que parece presentarse como una secuencia continua desde términos del Frasniense hasta el Viseense, deshaciendo el esquema estratigráfico anárquico que se traslucía en los trabajos anteriores.

En años posteriores se realizan las Hojas del Plan MAGNA, a Escala 1:50.000, que engloban gran parte de la zona de estudio, entre ellas, las de García Sansegundo et al. (1987a y b), Mira López et al. (1987) y Rodríguez Pevida et al. (1990). Los estudios paleontológicos del Devónico de las hojas, se deben al Dr. J.L. GarcíaAlcalde y colaboradores del Departamento de Paleontología de Oviedo, salvo para las hojas de Herrera del Duque y Almadén, que fueron realizados por el Dr. J.L. García-Alcalde y por uno de nosotros (M.V.P.A.). La publicación de estas hojas supone una puesta al día en las investigaciones sobre la geología regional de la zona.

Por último, dentro de un amplio trabajo sobre el preMesozoico peninsular, Gutiérrez Marco et al. (1990) han realizado una síntesis del Paleozoico post-Cámbrico en el autóctono de la Zona Centroibérica, con una pequeña síntesis estratigráfica sobre las series devónicas.

Posteriormente a esta fecha, y ya fuera de este estudio histórico, se publicaron varios trabajos debidos en su mayor parte a uno de nosotros (M.V.P.A.), realizados en el marco del trabajo de investigación de la tesis doctoral (Pardo Alonso, 1997); éstas, y otras referencias recientes, se pueden encontrar en una síntesis sobre el Devónico de la región, realizada por Pardo Alonso y García-Alcalde (1996). Para terminar, destacar que, en fecha reciente, se han elaborado las hojas del MAGNA correspondientes a Chillón (807) y Valdecaballeros (732), las dos últimas de esta zona que incluyen terrenos devónicos, y que están todavía pendientes de publicación.

\section{ANÁLISIS DE LAS CITAS DE ESPECIES DEVÓNICAS}

Una vez revisados los trabajos anteriores, se ha realizado un primer análisis sobre el desarrollo histórico del conocimiento paleontológico de la región. Solamente se han estudiado los trabajos que se centran en el área del presente trabajo; de este modo, se han excluido aquellos que revisan un grupo amplio de fósiles, del que hay representación en el área (Paeckelmann, 1942; Racheboeuf, 1981; House y Price, 1985). Para este análisis se ha utilizado como índice el número de especies citadas o descritas en cada publicación; este recuento se ha realizado exclusivamente para el material procedente de la región de Almadén, y sólo en una ocasión se han utilizado datos inéditos, con el fin de dar una visión más completa de un período que de otro modo aparecería vacío de aportaciones.

Las citas de especies en cada trabajo se han separado en tres categorías: nuevas especies descritas en la región, nuevas citas (especies que se citan por primera vez en la región, excluyendo las nuevas especies), y citas de especies anteriormente mencionadas (citas repetidas). El recuento de estas categorías ha sido elaborado sin tener en cuenta si las citas eran correctas o no, por lo que este dato deberá ser tomado con precaución a la hora de realizar el análisis sobre la adecuación taxonómica del trabajo. Esta reserva afecta sobre todo a la categoría de nuevas citas, que agrupa todas las determinaciones que suponen la entrada de un nombre específico no citado anteriormente en el área estudiada, aunque algunas de ellas sustituyen a otras anteriores.

Con los datos obtenidos del recuento, se han realizado dos diagramas de barras (Figs. 3 y 4). En el primero (Fig. 3) se han representado las aportaciones de cada trabajo al cómputo total de especies citadas en la región, representando en cada caso por separado las tres categorías señaladas. Esto ha permitido diferenciar cinco etapas respecto al conocimiento paleontológico del Devónico de la región; cuando ha sido posible, estos límites se han situado en hitos que ya han sido utilizados para la división en períodos del conocimiento geológico en España (Fernández de Castro, 1874; Mallada, 1897; Truyols, 1989; Gozalo, 1997); otras etapas son específicas de esta área, condicionadas por las circunstancias regionales. En la Fig. 4 se analizan de nuevo las citas de especies, esta vez representando el número total de especies citadas en cada período histórico; junto a ellas se ha representado cuántas de ese número total no se habían citado en períodos anteriores, es decir, cuántas de ellas fueron nuevas citas (incluyendo las especies nuevas) para la región. En el recuento se han tenido en cuenta los años de inicio y final de cada período, quedando en evidencia dos períodos donde no se produjeron aportaciones. 


\section{PROPUESTA DE DIVISIÓN EN PERÍODOS}

Con el análisis de la información reflejada en las Figs. 3 y 4 , se pueden distinguir siete grandes etapas para el área de Almadén:

Una Primera época, que abarcaría desde 1834 hasta 1873, y coincidiría con las etapas denominadas "Dirección General de Minas" (1825-1848) y "Primera Comisión del Mapa Geológico" (1849-1873) por Fernández de Castro (1874) y Mallada (1897). En ella se publicaron los primeros estudios regionales, incluyendo la primera monografía sobre la geología y la paleontología del Devónico de Almadén (Prado, 1855; Verneuil y Barrande, 1855) que marca un máximo en los tres índices de citas paleontológicas.

La segunda etapa, Reorganización de la Comisión del Mapa Geológico, se inicia en 1873 (Mallada, op. cit.), y termina en 1910, con la trasformación de la Comisión del Mapa en el Instituto Geológico de España (Gozalo, 1997). En esta época, la Comisión del Mapa Geológico de España inició el estudio sistemático y coordinado de la geología de nuestro país, descubriéndose materiales y fósiles del Devónico en áreas próximas a la estudiada, y realizándose mapas y bosquejos geológicos más detallados. Aunque para la paleontología de esta zona apenas supuso algunos añadidos a lo ya indicado por autores de la etapa anterior, es importante en este período la figura de Mallada, quien, aparte de realizar algunas de las nuevas aportaciones, actuó como recopilador y actualizador de todos los trabajos anteriores; de hecho, varias de sus nuevas citas que se reflejan en la Fig. 3, son en realidad una puesta al día o correcciones de citas anteriores, de acuerdo con los conceptos taxonómicos imperantes en la época.

La siguiente etapa abarca desde 1911 hasta la Guerra Civil, conocida en Cataluña como "Institucionalización de la Geología” (Truyols, 1989), y que se puede ampliar a todo el territorio nacional, y que aquí denominamos Primer tercio de siglo. El Instituto Geológico de España se vuelca más hacia el estudio de los yacimientos minerales y de la hidrogeología (Fallot, 1950), mientras que las investigaciones no mineras parecen quedar fuera de los objetivos de la investigación oficial en la región; un buen marcador del carácter de esta etapa es que en el año 1926, cuando se celebró en Madrid el XIV Congreso Geológico Internacional, el interés suscitado por esta área se debió únicamente a sus importantes yacimientos de mercurio. En lo que concierne a la estratigrafía y paleontología esta nueva etapa viene marcada por los trabajos de investigadores extranjeros; Groth separó por primera vez materiales y fósiles frasnienses y famenienses en el área, lo que fue la base del esquema estratigráfico y estructural que propuso Ciry (1936). Por otro lado, el trabajo de Drevermann (1909) sirvió como base al estudio de Müller (1929) sobre las faunas frasnienses, siguiente hito en la investigación paleontológica de la región.

El trabajo de Ciry (op cit. ) marca el final de la etapa y el inicio de un período vacío de aportaciones

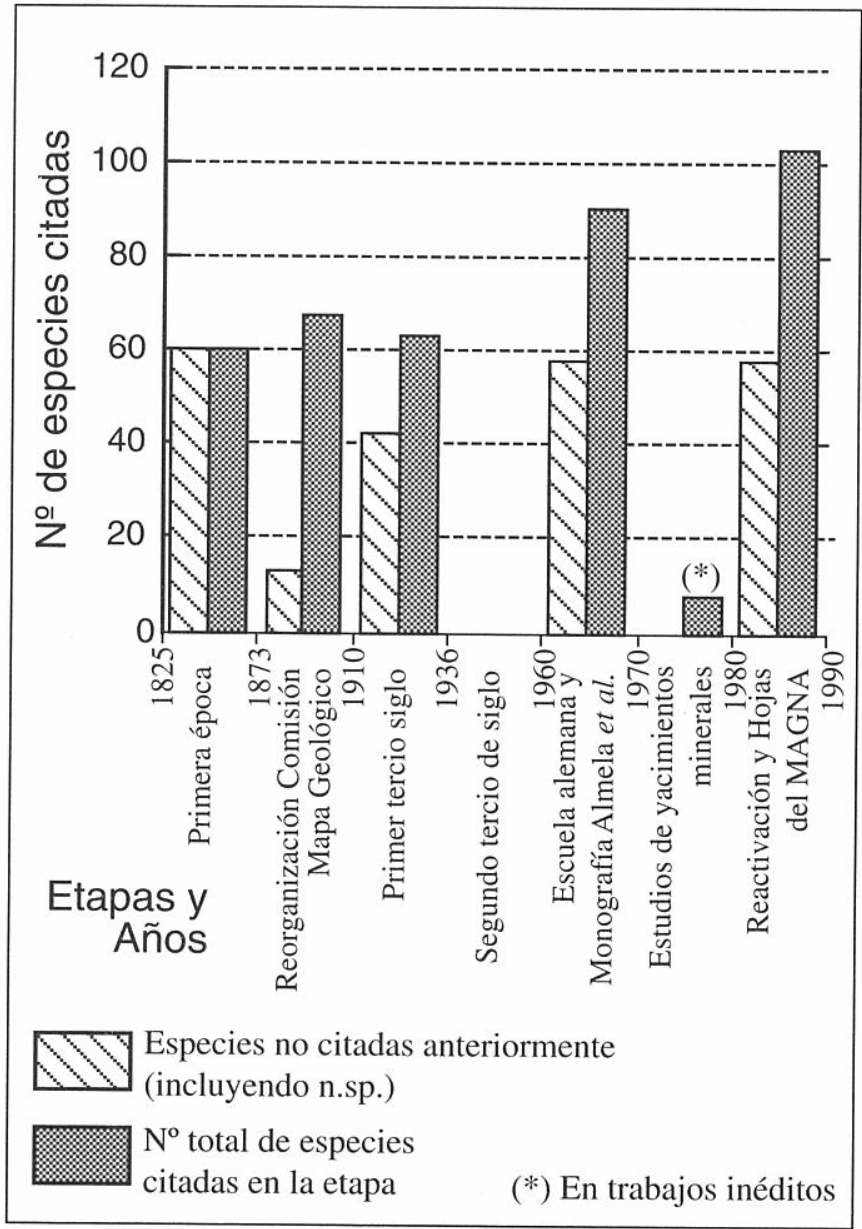

Figura 4. Distribución por etapas históricas de las especies fósiles del Devónico, citadas en el área de estudio, hasta 1990.

paleontológicas, que denominamos Segundo tercio de siglo, por coincidir aproximadamente con él (1936-1960). Este vacío no es una característica generalizada para toda España, ya que los estudios del Devónico continúan en otras zonas, y en la región de Almadén gana interés el estudio de los materiales y fósiles pre-devónicos. En esta época se realizaron diversas Tesis Doctorales por parte de alumnos de la escuela alemana de Münster, dirigidos por Franz Lotze, las cuales representan los antecedentes inmediatos de la siguiente etapa.

Este período, que denominamos Escuela alemana y Monografía de Almela et al., se iniciaría con los trabajos puntuales de Almela (1959) y Márquez Triguero (1961), para continuar con el estudio regional de Almela et al. (1962), y los trabajos de Puschmann a finales de los años sesenta y principios de los setenta. El volumen de aportaciones paleontológicas vuelve a ser importante, destacando especialmente el trabajo de Puschmann sobre Herrera del Duque; dos circunstancias explican el elevado volumen de nuevas citas: por un lado el estudio del Devónico Inferior de Herrera, muy rico en fósiles (mucho más que el de Almadén) y prácticamente virgen desde el trabajo de Verneuil y Barrande (1855); por otro, la identificación, por vez primera, de conodontos del 
Devónico en la región, que constituyen un volumen importante del total de nuevas citas. Este trabajo marca el final de la época, extensible, en gran parte, a otras áreas de España, que estuvo marcada por las aportaciones al conocimiento geológico regional de Lotze y colaboradores.

Entre 1970 y 1980, los trabajos sobre la zona que tocan temas paleontológicos, aunque son en gran parte de geología regional, tienen un interés preferente por los yacimientos mineros, esto se tradujo en un descenso brusco en el número de citas y de nuevas aportaciones al listado de especies. Por otro lado, durante el período tampoco mejoró el conocimiento de la estratigrafía del Devónico de la región. Estas características nos llevan a bautizar la etapa como: Estudios de yacimientos minerales.

La última época, que llamamos Reactivación y Hojas MAGNA se iniciaría en los años ochenta, y trajo consigo un nuevo impulso en los estudios paleontológicos y estratigráficos, centrados sobre todo en el área de Almadén. Mención especial merece Miguel Soler Sampere, de Minas de Almadén y Arrayanes, impulsor de la colaboración con las Universidades Autónoma de Barcelona y de Oviedo, que supuso la realización de varias Tesis de Licenciatura; una de ellas dio origen a las publicaciones de los trabajos de Pardo Alonso y GarcíaAlcalde y otros colaboradores de la Universidad de Oviedo, en 1984, que en conjunto, destacan en el gráfico (Fig. 3) como un nuevo máximo en citas de especies y descripción de nuevos taxones (circunstancia que no se daba desde el trabajo de Müller, 1929).

\section{AGRADECIMIENTOS}

Los autores quieren agradecer a D. Jaime Truyols su contribución a la búsqueda y elaboración de los datos que dieron lugar a este trabajo, y las gestiones que facilitaron nuestro acceso a la Biblioteca de la Real Academia de Ciencias. También queremos expresar nuestro agradecimiento a los Dres. Jenaro L. García-Alcalde y Juan Carlos Gutiérrez Marco, que con su detallada revisión del trabajo, contribuyeron a completarlo y mejorarlo, tanto en los aspectos formales como en los de fondo.

Este trabajo ha sido financiado en parte por el proyecto DGICYT PB94-1324 "Eventos geo-biológicos en el Devónico del Macizo Ibérico (España)" y es una contribución al proyecto I.G.C.P. n 421 "North Gondwana Mid-Palaeozoic Bioevent/Biogeography patterns in relation to crustal dynamics".

\section{BIBLIOGRAFÍA}

Almela, A. 1959. Esquema geológico de la zona de Almadén (Ciudad Real). Boletín Instituto Geológico y Minero de España, 70, 315-330.

Almela, A., Alvarado, M., Coma, J., Felgueroso, C. y Quintero, I. 1962. Estudio geológico de la región de Almadén. Boletín del Instituto Geológico y Minero de
España, 73, 197-327.

Anónimo. 1850. Apuntes sobre algunos fósiles hallados por Mr. de Verneuil en España. Revista Minera, 1, 95.

Ciry, G. 1936. Contribution à l'étude géologique de la région d'Almadén. Boletín de la Sociedad Española de Historia Natural, 36, 295-300.

Cortázar, D. de 1880. Reseña física y geológica de la provincia de Ciudad Real. Boletín de la Comisión del Mapa Geológico de España, 7, 289-329.

Díez-Balda, M.A., Vegas, R. and González-Lodeiro, F. 1990. Part IV. Central-Iberian Zone. 2.2. Structure. In: Pre-Mesozoic Geology of Iberia (Eds. R.D. Dallmeyer and E. Martínez-García). Springer-Verlag, Berlin, 172188.

Drevermann, F. 1909. Paläozoische Notizen. 2. Über Unterund Oberdevon bei Almadén in Spanien. Bericht der Senckenbergischen Naturforschunden Gesellschaft, 40, 76-78.

Ezquerra del Bayo, J. 1838. Apuntes geognósticos y mineros sobre una parte del mediodia de España. Anales de Minas, 1, 322-360.

Ezquerra del Bayo, J. 1854. Ensayo de una Descripcion general de la estructura geológica del terreno de España en la Península: seccion III. Memorias de la Real Academia de Ciencias de Madrid, 1, 161-184.

Ezquerra del Bayo, J. 1856. Ensayo de una Descripcion general de la estructura geológica del terreno de España en la Península: seccion IV. Memorias de la Real Academia de Ciencias de Madrid, 4, 115-155.

Fallot, P. 1950. Les progrés de la Géologie en Espagne depuis cent ans. Memorias de la Real Academia de Ciencias Exactas, Físicas y Naturales de Madrid, Serie 2, 11, 115-155.

Fernández de Castro, M. 1874. Notas para un estudio bibliográfico sobre los orígenes y estado actual del Mapa Geológico de España. Boletín de la Comisión del Mapa Geológico de España, 1, 1-152, 309-320.

García-Alcalde, J.L., Arbizu M.A., Pardo Alonso, M.V. y García-López, S. 1984. El límite Devónico-Carbonífero en el área de Guadalmez-Santa Eufemia (Provs. de Ciudad Real y Córdoba, Sierra Morena, España). I Congreso Español de Geología. Segovia, 1, 421-430.

García Sansegundo, J., Lorenzo Álvarez, S. y Ortega Gironés, E. 1987a. Mapa y memoria explicativa de la Hoja Siruela (781) del Mapa geológico de Epaña a escala 1:50.000. Instituto Geológico y Minero de España, 50 pp.

García Sansegundo, J., Lorenzo Álvarez, S. y Ortega Gironés, E. 1987b. Mapa y memoria explicativa de la Hoja Almadén (808) del Mapa geológico de España a escala 1:50.000. Instituto Geológico y Minero de España, 60 pp.

Gonzalo y Tarín, J. 1879. Reseña físico-geológica de la Provincia de Badajoz. Boletín de la Comisión del Mapa Geológico de España, 6, 389-412.

Gozalo, R. 1997. La geología española durante la Restauración. Actes de les IV Trobades d'Història de la Ciència i de la Tècnica, 143-152.

Groth, J. 1911. Sur le Primaire de la Sierra Morena. Comptes Rendus de l'Académie des Sciences Paris, 150, 231-232. 
Groth, J. 1914a. Les schistes à Goniatites de Guadalmez. Comptes Rendus de l'Académie des Sciences Paris, $\mathbf{1 5 8}$, 525-526.

Groth, J. 1914b. La Sierra Morena. Comptes Rendus de l'Académie des Sciences Paris, 158, 1722-1724.

Groth, J. 1914c. La tectonique de la sierra Morena. Comptes Rendus de l'Académie des Sciences Paris, 158, 19441946.

Gutiérrez Marco, J.C., San José, M.A. de and Pieren, A.P. 1990. 2.1.3. Post-Cambrian Palaeozoic Stratigraphy. In: Pre-Mesozoic Geology of Iberia (Eds. R.D. Dallmeyer and E. Martínez-García). Springer-Verlag, Berlin, 160171.

Hernández Sampelayo, P., Sierra y Yoldi, A. de, Puget, L.M. et Martí, C.M. 1926. Mines d'Almadén. XIVe Congrès International de Géologie. Excursión B-1, 36-41.

House, M.R. and Price, J.D. 1985. New Late Devonian genera and species of Tornoceratid Goniatites. Palaeontology, 28, 159-188.

Julivert, M., Fontboté, M., Ribeiro, A. y Conde, L. E. 1974. Memoria explicativa del Mapa Tectónico de la Península Ibérica y Baleares. E. 1:1.000.000. Instituto Geológico y Minero de España, Madrid, 1-113.

Julivert, M., Truyols, J. y Vergés, J. 1983. El Devónico del Macizo Ibérico. In: Geología de España, Libro Jubilar J. M. Ríos. Instituto Geológico y Minero de España, 1, 265-311.

Le Play, F. 1834. Observations sur l'Extremadure et le Nord de l'Andalousie et essai d'une carte geologique de cette contrée. Annales des Mines, 13, 297-500. Traduc. Cutoli y Lagoane, F. Descripción Geonóstica de Extremadura y Norte de Andalucia. Anales de Minas, 1841, 2, 143-196.

Luján, F. de. 1850. Estudios y observaciones geológicas relativas á terrenos que comprenden parte de la provincia de Badajoz, y de las de Sevilla, Toledo y Ciudad-Real, y cortes geológicos de estos terrenos. Memorias de la Real Academia de Ciencias de Madrid, 1 (1), 3-34.

Luján, F. de. 1851. Estudios y observaciones geológicas relativas á terrenos que comprenden parte de la provincia de Badajoz, y de las de Sevilla, Toledo y Ciudad-Real, y cortes geológicos de estos terrenos: parte $2^{\mathrm{a}}$. Memorias de la Real Academia de Ciencias de Madrid, 1 (2), 1-71.

Mallada, L. 1875. Sinopsis de las especies fósiles que se han encontrado en España. Tomo I, Sistemas Siluriano, Devoniano y Carbonífero. Boletín de la Comisión del Mapa Geológico de España, 2, 1-160.

Mallada, L. 1880. Reconocimiento geológico de la provincia de Córdoba. Boletín de la Comisión del Mapa Geológico de España, 7, 3-55.

Mallada, L. 1892. Catálogo general de las especies fósiles encontradas en España. Boletín de la Comisión del Mapa Geológico de España, 18, 1-253.

Mallada, L. 1897. Progresos de la Geología española durante el siglo XIX. Discurso de ingreso en la Academia de Ciencias exactas, físicas y naturales de Madrid, 89 pp.

Mallada, L. 1898. Explicación del Mapa Geológico de España. t. III , Sistemas Devoniano y Carbonífero. Memorias de la Comisión del Mapa Geológico de España, 21, 1-405.

Márquez Triguero, E. 1961. Estratigrafía del Paleozoico en la región del Río Guadalmez. Aportación a la Geología de Sierra Morena en la parte N del Valle de los Pedroches. Estudios Geológicos, 17, 187-201.

Mira López, M., Rodríguez Pevida, L.S. y Ortega Gironés, E. 1987. Mapa y memoria explicativa de la Hoja San Benito (834) del Mapa geológico de España a escala 1:50.000. Instituto Geológico y Minero de España, 52 pp.

Müller, W. 1929. Die Fauna der Frasnes-Stufe bei Almadén (Sierra Morena, Spanien). Abhandlungen der Senckenbergischen Naturforschenden Gesellschaft, 41 (5), 243-282.

Murchison, R.I. 1850. Notice on the Geological Structure of Spain, to explain an Outline General Map of the Peninsula. By M.E. de Verneuil. Report of the 20th. meeting British Association, 108-112.

Olivé Davó, A., Portero García, J.M., Álvaro López, A., Pieren Pidal, A., Aguilar, M.J., Dabrio, C. y Nozal, F. 1989. Memoria explicativa de la Hoja Herrera del Duque (756) del Mapa geológico de España a escala 1:50.000. Instituto Tecnológico y GeoMinero de España, $92 \mathrm{pp}$.

Paeckelmann, W. 1942. Beitrage zur kenntnis devonischer Spiriferen. Abhandlungen des Reichsamts für bodenforschung, N.F., 197, 1-188.

Pardo Alonso, M.V. 1997. El Devónico meridional de la Zona Centroibérica. Tesis Doctoral, Universidad de Oviedo, 472 pp. (inédito).

Pardo, M.V. y García-Alcalde J.L. 1984. Biostratigrafía del Devónico de la región de Almadén (Ciudad Real, España). Trabajos de Geología, Universidad de Oviedo, 14, 79-120.

Pardo Alonso, M.V. y García-Alcalde J.L. 1984. El Devónico de la región de Almadén (Ciudad Real, España). I Congreso Español de Geología. Segovia, 1, 473-482.

Pardo Alonso, M.V. y García-Alcalde, J.L. 1996. El Devónico de la Zona Centroibérica. Revista Española de Paleontología, $\mathbf{n}^{\mathbf{0}}$ extraordinario, 72-81.

Prado, C. de. 1855. Mémoire sur la géologie d'Almaden, d'une partie de la Sierra Morena et des montagnes de Tolède. Bulletin Société Géologique France, 2 ser., 12, 182-204, [1-24].

Puschmann, H. 1967a. Zum Problem der Schichtlücken im Devon der Sierra Morena (Spanien). Geologische Rundschau, 56, 528-542.

Puschmann, H. 1967b. Das Paläozoikum im Gebiet zwischen San Benito und Torrecampo (Sierra Morena/Spanien). Geologie Mijnbouw, 46, 383-391.

Puschmann, H. 1970a. Eine Paläogeographie des Devons auf der Iberischen Halbinsel. Zeitschript Deutschen Geologischen Geselleschaft, 120, 107-118.

Puschmann, H. 1970b. Das Paläozoikum der Nördlichen "Sierra Morena" am Beispiel der Mulde von Herrera del Duque (Spanien). Geologie, 19 (3), 309-329.

Racheboeuf, P.R. 1981. Chonétacés (Brachiopodes) Siluriens et Dévoniens du Sud-Ouest de l'Europe (Systématique-Phylogénie-BiostratigraphiePaléobiogéographie). Mémoires de la Société géologique et minéralogique de Bretagne, 27, 294 pp. 
Rodríguez Pevida, L.S., Mira López, M. y Ortega Gironés, E. 1990. Mapa y memoria explicativa de la Hoja Hinojosa del Duque (833) del Mapa geológico de España a escala 1:50.000. Instituto Tecnológico y GeoMinero de España, 58 pp.

Saupé, F. 1973. La géologie du gisement de mercure d'Almaden (province de Ciudad Real, Espagne). Mémoire Sciences Terre Nancy, 29, 342 pp.

Truyols, J. 1989. La Geologia catalana entre l'oficialitat i la iniciativa privada. Actes Universitaris, Publicacions de la Universitat de Barcelona, 1, 64 pp.

Vergés, J. 1983. Estudio del Complejo vulcano-sedimentario del Devónico y de la estructura de la terminación oriental del sinclinal de Almadén (Ciudad Real). In: Libro Jubilar J.M. Ríos, Instituto Geológico y Minero de España, 3, 215-229.

Verneuil, E. de et Barrande, J. 1855. Description des fossiles trouvés dans les terrains silurien et dévonien d'Almaden, d'une partie de la Sierra Morena et des Montagnes de Tolède. Bulletin de la Société Géologique de France, 2 Sér., 12, 964-1025, [25-86].

Verneuil, E. de et Collomb, E. 1853. Coup d'oeil sur la constitution géologique de quelques provinces de l'Espagne. Bulletin de la Société Géologique de France, 2 Sér., 10, 61-147. 